\title{
Successive iteration and positive solutions of a fractional boundary value problem on the half-line
}

\author{
Wenzhe Xie' ${ }^{1}$ Zhiguo Luo ${ }^{1}$ and Jing Xiao ${ }^{2 *}$
}

"Correspondence: xjazyh@163.com

${ }^{2}$ Department of Information Engineering, Guangdong Medica College, Dongguan, Guangdong 523808 , China

Full list of author information is

available at the end of the article

\begin{abstract}
In this paper, we study the existence of positive solutions for a nonlinear fractional boundary value problem on the half-line. Based on the monotone iterative technique, we obtain the existence of positive solutions of a fractional boundary value problem and establish iterative schemes for approximating the solutions. As application, an example is presented to illustrate the main results.
\end{abstract}

MSC: 34A08; 34A12; 34B40

Keywords: fractional boundary value problem; successive iteration; half-line; positive solutions

\section{Introduction}

The initial and boundary value problems for nonlinear fractional differential equations arise from the study of models of viscoelasticity, control, porous media, etc. [1, 2]. In the past few years, the existence and multiplicity of positive solutions for nonlinear fractional boundary value problems have been widely studied by many authors (see [3-11] and the references therein).

Most of these papers only considered the existence of positive solutions of various boundary value problems. Yet how can we find the solutions when their existence is known? Sun et al. [12] proved the existence of positive solutions for second-order $p$-Laplacian boundary value problems which are defined on finite intervals via the iterative technique. Liang and Zhang [13] investigated the existence of three positive solutions for the following $m$-point fractional boundary value problem on an infinite interval by means of the Leggett-Williams fixed point theorems on cones. In [14, 15], by employing the standard fixed point theorems and the monotone iterative technique, the authors deal with the existence of solutions for nonlinear fractional boundary value problems on an unbounded domain. Motivated by all the works above, we investigate the iteration and existence of positive solutions for the following fractional boundary value problems on the half-line:

$$
\left\{\begin{array}{l}
D_{0+}^{\alpha} u(t)+q(t) f(t, u(t))=0, \quad t \in(0, \infty) \\
u(0)=0, \quad \lim _{t \rightarrow \infty} D_{0+}^{\alpha-1} u(t)=\lambda \geq 0
\end{array}\right.
$$

where $1<\alpha<2, D_{0_{+}}^{\alpha}$ is the standard Riemann-Liouville fractional derivative. By applying monotone iterative techniques, we construct some successive iterative schemes to ap-

(c) 2013 Xie et al.: licensee Springer. This is an Open Access article distributed under the terms of the Creative Commons Attribution License (http://creativecommons.org/licenses/by/2.0), which permits unrestricted use, distribution, and reproduction in any medium, provided the original work is properly cited. 
proximate the solutions in this paper. They start off with a simple function and the zero function respectively, which is convenient for application.

Throughout this paper, we assume that the following conditions hold:

$\left(\mathrm{H}_{1}\right) f \in C([0, \infty) \times[0, \infty),[0, \infty)), f(t, 0) \not \equiv 0$ on any subinterval of $[0, \infty)$ and when $u$ is bounded, $f\left(t,\left(1+t^{\alpha-1}\right) u\right)$ is bounded on $[0, \infty)$;

$\left(\mathrm{H}_{2}\right) q(t):[0, \infty) \rightarrow[0, \infty)$ is not identical zero on any closed subinterval of $[0, \infty)$, and $0<\int_{0}^{\infty} q(s) d s<\infty$.

\section{Preliminaries}

We need the following definitions and lemmas that will be used to prove our main results.

Definition 2.1 [1] The integral

$$
I_{0+}^{\alpha} f(t)=\frac{1}{\Gamma(\alpha)} \int_{0}^{t} \frac{f(s)}{(t-s)^{1-\alpha}} d s, \quad t>0,
$$

where $\alpha>0$, is called the Riemann-Liouville fractional integral of order $\alpha$ and $\Gamma(\alpha)$ is the Euler gamma function defined by $\Gamma(\alpha)=\int_{0}^{\infty} t^{\alpha-1} e^{-t} d t, \alpha>0$.

Definition 2.2 [2] For a function $f(x)$ given in the interval $[0, \infty)$, the expression

$$
D_{0+}^{\alpha} f(t)=\frac{1}{\Gamma(\alpha)}\left(\frac{d}{d t}\right)^{n} \int_{0}^{t} \frac{f(s)}{(t-s)^{\alpha-n+1}} d s,
$$

where $n=[\alpha]+1,[\alpha]$ denotes the integer part of number $\alpha$, is called the Riemann-Liouville fractional derivative of order $\alpha$.

Lemma 2.1 [2] Let $\alpha>0$ and $u \in C(0, \infty) \cap L(0, \infty)$. Then the fractional differential equation

$$
D_{0+}^{\alpha} u(t)=0
$$

has

$$
u(t)=c_{1} t^{\alpha-1}+c_{2} t^{\alpha-2}+\cdots+c_{n} t^{\alpha-n}, \quad c_{i} \in R, i=0,1, \ldots, n, n=[\alpha]+1
$$

as a unique solution.

Lemma 2.2 [2] Assume that $u(t) \in C(0, \infty) \cap L(0, \infty)$ with a fractional derivative of order $\alpha>0$ that belongs to $C(0, \infty) \cap L(0, \infty)$. Then

$$
I_{0+}^{\alpha} D_{0+}^{\alpha} u(t)=u(t)+c_{1} t^{\alpha-1}+c_{2} t^{\alpha-2}+\cdots+c_{n} t^{\alpha-n},
$$

for some $c_{i} \in R, i=0,1, \ldots, n, n=[\alpha]+1$.

Lemma 2.3 Let $g \in C[0, \infty)$, then the fractional boundary value problem

$$
\left\{\begin{array}{l}
D_{0+}^{\alpha} u(t)+g(t)=0, \quad t \in(0, \infty), 1<\alpha<2 \\
u(0)=0, \quad \lim _{t \rightarrow \infty} D_{0+}^{\alpha-1} u(t)=\lambda \geq 0
\end{array}\right.
$$


has a unique solution

$$
u(t)=\int_{0}^{\infty} G(t, s) g(s) d s+\frac{\lambda}{\Gamma(\alpha)} t^{\alpha-1}
$$

where

$$
G(t, s)=\frac{1}{\Gamma(\alpha)} \begin{cases}t^{\alpha-1}-(t-s)^{\alpha-1}, & 0 \leq s \leq t<\infty \\ t^{\alpha-1}, & 0 \leq t \leq s<\infty\end{cases}
$$

Proof By Lemma 2.2, the solution of (2.1) can be written as

$$
u(t)=c_{1} t^{\alpha-1}+c_{2} t^{\alpha-2}-\int_{0}^{t} \frac{(t-s)^{\alpha-1}}{\Gamma(\alpha)} g(s) d s .
$$

From $u(0)=0$, we know that $c_{2}=0$.

Together with $\lim _{t \rightarrow \infty} D_{0+}^{\alpha-1} u(t)=\lambda \geq 0$, we have

$$
c_{1}=\frac{\lambda+\int_{0}^{\infty} g(s) d s}{\Gamma(\alpha)} .
$$

Therefore, the unique solution of fractional boundary value problem (2.1) is

$$
\begin{aligned}
u(t) & =-\int_{0}^{t} \frac{(t-s)^{\alpha-1}}{\Gamma(\alpha)} g(s) d s+\int_{0}^{\infty} \frac{t^{\alpha-1}}{\Gamma(\alpha)} g(s) d s+\frac{\lambda}{\Gamma(\alpha)} t^{\alpha-1} \\
& =\int_{0}^{t} \frac{t^{\alpha-1}-(t-s)^{\alpha-1}}{\Gamma(\alpha)} g(s) d s+\int_{t}^{\infty} \frac{t^{\alpha-1}}{\Gamma(\alpha)} g(s) d s+\frac{\lambda}{\Gamma(\alpha)} t^{\alpha-1} \\
& =\int_{0}^{\infty} G(t, s) g(s) d s+\frac{\lambda}{\Gamma(\alpha)} t^{\alpha-1} .
\end{aligned}
$$

The proof is complete.

Lemma 2.4 The function $G(t, s)$ defined by (2.2) satisfies the following:

(1) $G(t, s)$ is a continuous function and $G(t, s) \geq 0$ for $(t, s) \in[0, \infty) \times[0, \infty)$;

(2) $G(t, s) \leq \frac{t^{\alpha-1}}{\Gamma(\alpha)}, \frac{G(t, s)}{1+t^{\alpha-1}} \leq \frac{1}{\Gamma(\alpha)}$ for $(t, s) \in[0, \infty) \times[0, \infty)$.

The proof is easy, so we omit it here.

In this paper, we use the following space $E$, which is denoted by

$$
E=\left\{u \in C[0, \infty): \sup _{0 \leq t<\infty} \frac{|u(t)|}{1+t^{\alpha-1}}<\infty\right\}
$$

to study fractional boundary value problem (1.1). From [16], we know that $E$ is a Banach space equipped with the norm $\|u\|=\sup _{0 \leq t<\infty} \frac{|u(t)|}{1+t^{\alpha-1}}<\infty$. Define the cone $P \subset E$ by

$$
P=\{u \in E: u(t) \geq 0, t \in[0, \infty)\},
$$

and an integral operator $T: P \rightarrow E$ by

$$
\operatorname{Tu}(t)=\int_{0}^{\infty} G(t, s) q(s) f(s, u(s)) d s+\frac{\lambda}{\Gamma(\alpha)} t^{\alpha-1}, \quad 0 \leq t<\infty,
$$

where $G(t, s)$ is defined by $(2.2)$. 
Lemma 2.5 [16] Let $V=\{u \in E:\|u\|<l\}(l>0), V_{1}=\left\{\frac{u(t)}{1+t^{\alpha-1}}: u \in V\right\}$. If $V_{1}$ is equicontinuous on any compact intervals of $[0, \infty)$ and equiconvergent at infinity, then $V$ is relatively compact on $E$.

Remark 2.5 $V_{1}$ is called equiconvergent at infinity if and only if for all $\varepsilon>0$, there exists $v(\varepsilon)>0$ such that for all $u \in V_{1}, t_{1}, t_{2} \geq v$, the following holds:

$$
\left|\frac{u\left(t_{1}\right)}{1+t_{1}^{\alpha-1}}-\frac{u\left(t_{2}\right)}{1+t_{2}^{\alpha-1}}\right|<\varepsilon
$$

Lemma 2.6 Let $\left(\mathrm{H}_{1}\right)$ and $\left(\mathrm{H}_{2}\right)$ hold, then $T: P \rightarrow P$ is completely continuous.

Proof First, it is easy to check that $T: P \rightarrow P$ is well defined. Now, we prove that $T$ is continuous and compact respectively. Let $u_{n} \rightarrow u$ as $n \rightarrow \infty$ in $P$, then there exists $r_{0}$ such that $\sup _{n \in N \backslash\{0\}}\left\|u_{n}\right\|<r_{0}$. Let $B_{r_{0}}=\sup \left\{f\left(t,\left(1+t^{\alpha-1}\right) u\right) \mid(t, u) \in[0, \infty) \times\left[0, r_{0}\right]\right\}$. By $\left(\mathrm{H}_{2}\right)$, we have

$$
\int_{0}^{\infty} q(s) f(s, u(s)) d s \leq B_{r_{0}} \int_{0}^{\infty} q(s) d s<\infty
$$

Then by the Lebesgue dominated convergence theorem and the continuity of $f$, we can get

$$
\int_{0}^{\infty} q(s) f\left(s, u_{n}(s)\right) d s \rightarrow \int_{0}^{\infty} q(s) f(s, u(s)) d s \quad \text { as } n \rightarrow \infty .
$$

Therefore, we have

$$
\begin{aligned}
\left\|T u_{n}-T u\right\| & =\sup _{0 \leq t<\infty}\left|\int_{0}^{\infty} \frac{G(t, s)}{1+t^{\alpha-1}} q(s)\left[f\left(s, u_{n}(s)\right)-f(s, u(s))\right] d s\right| \\
& \leq \frac{1}{\Gamma(\alpha)}\left|\int_{0}^{\infty} q(s) f\left(s, u_{n}(s)\right) d s-\int_{0}^{\infty} q(s) f(s, u(s)) d s\right| \rightarrow 0, \quad n \rightarrow \infty .
\end{aligned}
$$

Then $T$ is continuous.

Let $\Omega$ be any bounded subset of $P$. Then there exists $r>0$ such that $\|u\| \leq r$ for any $u \in \Omega$. Let $B_{r}=\sup \left\{f\left(t,\left(1+t^{\alpha-1}\right) u\right) \mid(t, u) \in[0, \infty) \times[0, r]\right\}$, therefore, from Lemma 2.4, we have

$$
\begin{aligned}
\|T u\| & =\sup _{0 \leq t<\infty} \frac{1}{1+t^{\alpha-1}}\left|\int_{0}^{\infty} G(t, s) q(s) f(s, u(s)) d s+\frac{\lambda}{\Gamma(\alpha)} t^{\alpha-1}\right| \\
& \leq \frac{B_{r}}{\Gamma(\alpha)} \int_{0}^{\infty} q(s) d s+\frac{\lambda}{\Gamma(\alpha)}<\infty .
\end{aligned}
$$

So, $T \Omega$ is bounded. Moreover, for any $T \in(0, \infty)$ and $t_{1}, t_{2} \in[0, T]$, without loss of generality, we may assume that $t_{2}>t_{1}$, we have

$$
\begin{aligned}
& \left|\frac{T u\left(t_{1}\right)}{1+t_{1}^{\alpha-1}}-\frac{T u\left(t_{2}\right)}{1+t_{2}^{\alpha-1}}\right| \\
& \quad \leq \int_{0}^{\infty}\left|\frac{G\left(t_{1}, s\right)}{1+t_{1}^{\alpha-1}}-\frac{G\left(t_{2}, s\right)}{1+t_{2}^{\alpha-1}}\right| q(s) f(s, u(s)) d s+\frac{\lambda}{\Gamma(\alpha)}\left|t_{1}^{\alpha-1}-t_{2}^{\alpha-1}\right|
\end{aligned}
$$




$$
\begin{aligned}
\leq & \int_{0}^{\infty}\left|\frac{G\left(t_{1}, s\right)}{1+t_{1}^{\alpha-1}}-\frac{G\left(t_{2}, s\right)}{1+t_{1}^{\alpha-1}}\right| q(s) f(s, u(s)) d s \\
& +\int_{0}^{\infty}\left|\frac{G\left(t_{2}, s\right)}{1+t_{1}^{\alpha-1}}-\frac{G\left(t_{2}, s\right)}{1+t_{2}^{\alpha-1}}\right| q(s) f(s, u(s)) d s+\frac{\lambda}{\Gamma(\alpha)}\left|t_{1}^{\alpha-1}-t_{2}^{\alpha-1}\right| .
\end{aligned}
$$

On the other hand, we have

$$
\begin{aligned}
\int_{0}^{\infty} & \left|\frac{G\left(t_{1}, s\right)}{1+t_{1}^{\alpha-1}}-\frac{G\left(t_{2}, s\right)}{1+t_{1}^{\alpha-1}}\right| q(s) f(s, u(s)) d s \\
\leq & \int_{0}^{t_{1}}\left|\frac{G\left(t_{1}, s\right)}{1+t_{1}^{\alpha-1}}-\frac{G\left(t_{2}, s\right)}{1+t_{1}^{\alpha-1}}\right| q(s) f(s, u(s)) d s \\
& +\int_{t_{1}}^{t_{2}}\left|\frac{G\left(t_{1}, s\right)}{1+t_{1}^{\alpha-1}}-\frac{G\left(t_{2}, s\right)}{1+t_{1}^{\alpha-1}}\right| q(s) f(s, u(s)) d s \\
& +\int_{t_{2}}^{\infty}\left|\frac{G\left(t_{1}, s\right)}{1+t_{1}^{\alpha-1}}-\frac{G\left(t_{2}, s\right)}{1+t_{1}^{\alpha-1}}\right| q(s) f(s, u(s)) d s \\
\leq & \frac{B_{r}}{\Gamma(\alpha)} \int_{0}^{t_{1}} \frac{\left[t_{2}^{\alpha-1}-t_{1}^{\alpha-1}\right]+\left[\left(t_{2}-s\right)^{\alpha-1}-\left(t_{1}-s\right)^{\alpha-1}\right]}{1+t_{1}^{\alpha-1}} q(s) d s \\
& +\frac{B_{r}}{\Gamma(\alpha)} \int_{t_{1}}^{t_{2}} \frac{\left[t_{2}^{\alpha-1}-t_{1}^{\alpha-1}\right]+\left(t_{2}-s\right)^{\alpha-1}}{1+t_{1}^{\alpha-1}} q(s) d s+\frac{B_{r}}{\Gamma(\alpha)} \int_{t_{2}}^{\infty} \frac{t_{2}^{\alpha-1}-t_{1}^{\alpha-1}}{1+t_{1}^{\alpha-1}} q(s) d s .
\end{aligned}
$$

Similarly, we have

$$
\begin{aligned}
& \int_{0}^{\infty}\left|\frac{G\left(t_{2}, s\right)}{1+t_{1}^{\alpha-1}}-\frac{G\left(t_{2}, s\right)}{1+t_{2}^{\alpha-1}}\right| q(s) f(s, u(s)) d s \rightarrow 0 \quad \text { uniformly as } t_{1} \rightarrow t_{2}, \\
& \frac{\lambda}{\Gamma(\alpha)}\left|t_{1}^{\alpha-1}-t_{2}^{\alpha-1}\right| \rightarrow 0 \quad \text { uniformly as } t_{1} \rightarrow t_{2} .
\end{aligned}
$$

Hence, $T \Omega$ is locally equicontinuous on $[0, \infty)$.

Next, we show that $T: P \rightarrow E$ is equiconvergent at $\infty$. For any $u \in \Omega$, we have

$$
\int_{0}^{\infty} q(s) f(s, u(s)) d s<\infty
$$

and

$$
\begin{aligned}
\lim _{t \rightarrow \infty}\left|\frac{T u(t)}{1+t^{\alpha-1}}\right| & =\lim _{t \rightarrow \infty} \frac{1}{1+t^{\alpha-1}} \int_{0}^{\infty} G(t, s) q(s) f(s, u(s)) d s+\lim _{t \rightarrow \infty} \frac{\lambda}{\Gamma(\alpha)} \cdot \frac{t^{\alpha-1}}{1+t^{\alpha-1}} \\
& =\frac{\lambda}{\Gamma(\alpha)}<\infty .
\end{aligned}
$$

Hence, $T \Omega$ is equiconvergent at infinity. By using Lemma 2.5 , we obtain that $T: P \rightarrow P$ is completely continuous. The proof is complete.

\section{Main results}

We will prove the following existence results.

Theorem 3.1 Assume that $\left(\mathrm{H}_{1}\right)$ and $\left(\mathrm{H}_{2}\right)$ hold, and there exists $a>\frac{2 \lambda}{\Gamma(\alpha)}$ such that:

$\left(\mathrm{H}_{3}\right) f\left(t, u_{1}\right) \leq f\left(t, u_{2}\right)$ for any $0 \leq t<\infty, 0 \leq u_{1} \leq u_{2}$;

$\left(\mathrm{H}_{4}\right) f\left(t,\left(1+t^{\alpha-1}\right) u\right) \leq \frac{a \Gamma(\alpha)}{2 \int_{0}^{\infty} q(s) d s},(t, u) \in[0, \infty) \times[0, a]$. 
Then fractional boundary value problem (1.1) has two positive solutions $w^{*}$ and $v^{\prime \prime}$ on $[0, \infty)$, satisfying $0 \leq\left\|w^{*}\right\| \leq a, 0 \leq\left\|v^{*}\right\| \leq a, 0<v^{*} \leq w^{*}$, and

$$
\lim _{n \rightarrow \infty} w_{n}=\lim _{n \rightarrow \infty} T^{n} w_{0}=w^{\prime \prime}, \quad \lim _{n \rightarrow \infty} v_{n}=\lim _{n \rightarrow \infty} T^{n} v_{0}(t)=v^{*},
$$

where

$$
w_{0}(t)=\left(\frac{a}{2}+\frac{\lambda}{\Gamma(\alpha)}\right) t^{\alpha-1}, \quad v_{0}(t)=0, \quad t \in[0, \infty)
$$

Proof By Lemma 2.6, we know that $T: P \rightarrow P$ is completely continuous. For any $u_{1}, u_{2} \in P$ with $u_{1} \leq u_{2}$, from the definition of $T$ and $\left(\mathrm{H}_{3}\right)$, we can easily get that $T u_{1} \leq T u_{2}$. We denote

$$
\overline{P_{a}}=\{u \in P:\|u\| \leq a\} .
$$

Then, in what follows, we first prove that $T: \overline{P_{a}} \rightarrow \overline{P_{a}}$.

If $u \in \overline{P_{a}}$, then $\|u\| \leq a$, that is, $0 \leq \frac{u(t)}{1+t^{\alpha-1}} \leq a, 0 \leq t<\infty$. Then assumption $\left(\mathrm{H}_{4}\right)$ implies $f(t, u(t)) \leq \frac{a \Gamma(\alpha)}{2 \int_{0}^{\infty} q(s) d s}$ for all $(t, u) \in[0, \infty) \times[0, a]$, together with (2.3), $\left(\mathrm{H}_{3}\right)$, we get

$$
\begin{aligned}
\|T u\| & =\sup _{0 \leq t<\infty}\left|\int_{0}^{\infty} \frac{G(t, s)}{1+t^{\alpha-1}} q(s) f(s, u(s)) d s+\frac{\lambda}{\Gamma(\alpha)} \cdot \frac{t^{\alpha-1}}{1+t^{\alpha-1}}\right| \\
& \leq \frac{\int_{0}^{\infty} q(s) d s}{\Gamma(\alpha)} \cdot \frac{a \Gamma(\alpha)}{2 \int_{0}^{\infty} q(s) d s}+\frac{\lambda}{\Gamma(\alpha)} \\
& <\frac{a}{2}+\frac{a}{2}=a .
\end{aligned}
$$

Hence, we have proved that $T: \overline{P_{a}} \rightarrow \overline{P_{a}}$.

Let $w_{0}(t)=\left(\frac{a}{2}+\frac{\lambda}{\Gamma(\alpha)}\right) t^{\alpha-1}, 0 \leq t<\infty$, then $w_{0}(t) \in \overline{P_{a}}$. Let $w_{1}=T w_{0}, w_{2}=T^{2} w_{0}$; then by Lemma 2.6, we have that $w_{1} \in \overline{P_{a}}$ and $w_{2} \in \overline{P_{a}}$. We denote $w_{n+1}=T w_{n}=T^{n} w_{0}, n=0,1, \ldots$ Since $T: \overline{P_{a}} \rightarrow \overline{P_{a}}$, we have that $w_{n} \in T\left(\overline{P_{a}}\right) \subset \overline{P_{a}}, n=1,2, \ldots$ It follows from the complete continuity of $T$ that $\left\{w_{n}\right\}_{n=1}^{\infty}$ is a sequentially compact set.

By $(2.3)$ and $\left(\mathrm{H}_{4}\right)$, we get

$$
\begin{aligned}
w_{1}(t) & =\int_{0}^{\infty} G(t, s) q(s) f\left(s, w_{0}(s)\right) d s+\frac{\lambda}{\Gamma(\alpha)} t^{\alpha-1} \\
& \leq \frac{a \Gamma(\alpha)}{2 \int_{0}^{\infty} q(s) d s} \cdot \int_{0}^{\infty} \frac{t^{\alpha-1}}{\Gamma(\alpha)} q(s) d s+\frac{\lambda}{\Gamma(\alpha)} t^{\alpha-1} \\
& =\left(\frac{a}{2}+\frac{\lambda}{\Gamma(\alpha)}\right) t^{\alpha-1} \\
& =w_{0}(t) .
\end{aligned}
$$

So, by $\left(\mathrm{H}_{3}\right)$, we have

$$
w_{2}(t)=T w_{1}(t) \leq T w_{0}(t) \leq w_{1}(t), \quad 0 \leq t<\infty
$$


By induction, we get

$$
w_{n+1} \leq w_{n}, \quad 0 \leq t<\infty, n=0,1,2, \ldots
$$

Thus, there exists $w^{*} \in \overline{P_{a}}$ such that $w_{n} \rightarrow w^{*}$ as $n \rightarrow \infty$. Applying the continuity of $T$ and $w_{n+1}=T w_{n}$, we get that $T w^{*}=w^{*}$.

Let $v_{0}(t)=0,0 \leq t<\infty$; then $v_{0}(t) \in \overline{P_{a}}$. Let $v_{1}=T v_{0}, v_{2}=T^{2} v_{0}$; then by Lemma 2.6, we know that $v_{1} \in \overline{P_{a}}$ and $v_{2} \in \overline{P_{a}}$. We denote $v_{n+1}=T v_{n}=T^{n} v_{0}, n=0,1,2, \ldots$. Since $T: \overline{P_{a}} \rightarrow$ $\overline{P_{a}}$, we have $v_{n} \in T\left(\overline{P_{a}}\right) \subset \overline{P_{a}}, n=1,2, \ldots$ It follows from the complete continuity of $T$ that $\left\{v_{n}\right\}_{n=1}^{\infty}$ is a sequentially compact set.

Since $v_{1}=T v_{0} \in \overline{P_{a}}$, we have

$$
v_{1}(t)=\left(T v_{0}\right)(t)=(T 0)(t) \geq 0, \quad 0 \leq t<\infty
$$

So, we have

$$
v_{2}(t)=\left(T v_{1}\right)(t) \geq\left(T v_{0}\right)(t)=v_{1}(t), \quad 0 \leq t<\infty
$$

By induction, we get

$$
v_{n+1} \geq v_{n}, \quad 0 \leq t<\infty, n=0,1,2, \ldots
$$

Thus, there exists $v^{*} \in \overline{P_{a}}$ such that $v_{n} \rightarrow v^{*}$ as $n \rightarrow \infty$. Applying the continuity of $T$ and $v_{n+1}=T v_{n}$, we get $T v^{*}=v^{*}$.

Since $w_{0}(t)=\left(\frac{a}{2}+\frac{\lambda}{\Gamma(\alpha)}\right) t^{\alpha-1} \geq v_{0}=0$, and the operator $T$ is increasing, then

$$
w_{1}=T w_{0} \geq T v_{0}=v_{1}
$$

by induction, we have

$$
w_{n} \geq v_{n}, \quad 0 \leq t<\infty, n=0,1,2, \ldots
$$

Together with (3.1), (3.2), we obtain

$$
v_{0} \leq v_{1} \leq \cdots \leq v_{n} \leq v_{n+1} \leq \cdots \leq w_{n+1} \leq w_{n} \leq \cdots \leq w_{0} .
$$

If $f(t, 0) \not \equiv 0,0 \leq t<\infty$, then the zero function is not the solution of fractional boundary value problem (1.1). Thus, $v^{*}$ is a positive solution of fractional boundary value problem (1.1).

It is well known that each fixed point of $T$ in $P$ is a solution of fractional boundary value problem (1.1). Hence, $w^{*}$ and $v^{*}$ are two positive solutions on $[0, \infty)$ of fractional boundary value problem (1.1), satisfying $0<T v_{0} \leq v^{*} \leq w^{*}$.

\section{An example}

Example 4.1 Consider the boundary value problem of the fractional differential equation.

$$
\left\{\begin{array}{l}
D_{0+}^{\frac{3}{2}} u(t)+e^{-t} f(t, u(t))=0, \quad t \in(0, \infty) \\
u(0)=0, \quad \lim _{t \rightarrow \infty} D_{0+}^{\frac{1}{2}} u(t)=0
\end{array}\right.
$$


where

$$
f(t, u(t))= \begin{cases}|\cos t|+\left(\frac{u}{1+\sqrt{t}}\right)^{3}, & 0 \leq t<\infty, 0 \leq u \leq 2 \\ |\cos t|+\left(\frac{2}{1+\sqrt{t}}\right)^{3}, & 0 \leq t<\infty, u \geq 2\end{cases}
$$

In this case, $\alpha=\frac{3}{2}, q(t)=e^{-t}, \lambda=0, \Gamma\left(\frac{3}{2}\right) \approx 0.886$. It is clear that $\left(\mathrm{H}_{1}\right)-\left(\mathrm{H}_{3}\right)$ hold. Select $a=30$, by direct calculation, we can obtain

$$
\begin{aligned}
\int_{0}^{\infty} q(s) d s=\int_{0}^{\infty} e^{-s} d s & =1, \\
f\left(t,\left(1+t^{\frac{1}{2}}\right) u\right) & \leq 1+2^{3}=9<\frac{a \Gamma(\alpha)}{2 \int_{0}^{\infty} q(s) d s} \approx \frac{30 \times 0.886}{2} \\
& =13.29, \quad t \in[0, \infty), u \in[0,30] .
\end{aligned}
$$

The conditions in Theorem 3.1 are all satisfied. Therefore, fractional boundary value problem (4.1) has two positive solutions.

\section{Competing interests}

The authors declare that they have no competing interests.

\section{Authors' contributions}

WX and JX conceived of the studies, and drafted the manuscript. ZL participated in the discussion. All authors read and approved the final manuscript.

\section{Author details}

${ }^{1}$ Department of Mathematics, Hunan Normal University, Changsha, Hunan 410081, China. ${ }^{2}$ Department of Information Engineering, Guangdong Medical College, Dongguan, Guangdong 523808, China

\section{Acknowledgements}

This work is supported by the National Nature Science Foundation of P.R. China (10871063), partially supported by the National Nature Science Foundation of P.R. China (61170320), the Foundation of Guangdong Natural Science (S2011040002981), and the Nature Science Foundation of Guangdong Medical College (B2012053).

Received: 17 April 2013 Accepted: 28 June 2013 Published: 12 July 2013

\section{References}

1. Samko, SG, Kilbas, AA, Marichev, Ol: Fractional Integrals and Derivatives: Theory and Applications. Gordon \& Breach, Yverdon (1993)

2. Podlubny, I: Fractional Differential Equations. Mathematics in Sciences and Engineering, vol. 198. Academic Press, San Diego (1999)

3. Nieto, JJ: Maximum principles for fractional differential equations derived from Mittag-Leffler functions. Appl. Math. Lett. 23, 1248-1251 (2010)

4. Zhang, X, Ge, W: Unbounded solutions for a fractional boundary value problem on the infinite interval. Appl. Math. Lett. 109, 495-505 (2010)

5. Goodrich, CS: Existence of a positive solution to a class of fractional differential equations. Appl. Math. Lett. 23, 1050-1055 (2010)

6. Alves, E, Ma, TF, Pelicer, ML: Monotone positive solutions for a fourth order equation with nonlinear boundary conditions. Nonlinear Anal. 71, 3834-3841 (2009)

7. Ma, D, Du, Z, Ge, W: Existence and iteration of monotone positive solutions for multipoint boundary value problem with $p$-Laplacian operator. Comput. Math. Appl. 50, 729-739 (2005)

8. Chen, TY, Liu, WB: An anti-periodic boundary value problem for the fractional differential equation with a $p$-Laplacian operator. Appl. Math. Lett. 25, 1671-1675 (2012)

9. Ahmad, B, Nieto, JJ: Riemann-Liouville fractional differential equations with fractional boundary conditions. Fixed Point Theory 13, 329-336 (2012)

10. Arara, A, Benchohra, M, Hamidi, N, Nieto, JJ: Fractional order differential equations on an unbounded domain Nonlinear Anal. 72, 580-586 (2010)

11. Yang, L, Zhang, WG, Liu, XP: A sufficient condition for the existence of a positive solution for a nonlinear fractional differential equation with the Riemann-Liouville derivative. Appl. Math. Lett. 25, 1986-1992 (2012)

12. Sun, B, Zhao, X, Ge, W: Successive iteration and positive solutions for a $p$-Laplacian multipoint boundary value problem. ANZIAM J. 49, 551-560 (2008) 
13. Liang, S, Zhang, J: Existence of three positive solutions of $m$-point boundary value problems for some nonlinear fractional differential equations on an infinite interval. Comput. Math. Appl. 61, 3343-3354 (2011)

14. Zhang, L, Wang, G, Ahmad, B, Agarwal, RP: Nonlinear fractional integro-differential equations on unbounded domains in a Banach space. J. Comput. Appl. Math. 249, 51-56 (2013)

15. Wang, G, Ahmad, B, Zhang, L: A coupled system of nonlinear fractional differential equations with multi-point fractional boundary conditions on an unbounded domain. Abstr. Appl. Anal. 2012, Article ID 248709 (2012)

16. Liu, YS: Existence and unboundedness of positive solutions for singular boundary value problem on half-line. Appl. Math. Comput. 144, 543-556 (2003)

doi:10.1186/1687-1847-2013-210

Cite this article as: Xie et al.: Successive iteration and positive solutions of a fractional boundary value problem on the half-line. Advances in Difference Equations 2013 2013:210

Submit your manuscript to a SpringerOpen ${ }^{\odot}$ journal and benefit from:

- Convenient online submission

- Rigorous peer review

- Immediate publication on acceptance

- Open access: articles freely available online

- High visibility within the field

- Retaining the copyright to your article 